\title{
Timing and Clinical Features of Spontaneous Decrease in Size of Small Pancreatic Cystic Lesions without High-Risk Stigmata
}

\author{
Hyun Woo Lee ${ }^{1}$, Sung Koo Lee ${ }^{2}$, Jae Hyuck Jun ${ }^{2}$, Tae Jun Song ${ }^{2}$, Do Hyun Park ${ }^{2}$, Sang Soo Lee ${ }^{2}$, Dong-Wan Seo ${ }^{2}$, and \\ Myung-Hwan $\mathrm{Kim}^{2}$ \\ ${ }^{I}$ Digestive Disease Center and Research Institute, Department of Internal Medicine, Soonchunhyang University School of Medicine, Bucheon, \\ and ${ }^{2}$ Department of Internal Medicine, Asan Medical Center, University of Ulsan College of Medicine, Seoul, Korea
}

Background/Aims: The natural history of spontaneous decrease in the size of pancreatic cystic lesions (PCLs) without high-risk stigmata is under investigation. This study aimed to investigate the timing of spontaneous decrease in the size of PCLs without high-risk stigmata and to identify the characteristics associated with their complete resolution. Methods: From 2000 to 2016, patients with spontaneous decreases in PCL size on computed tomography (CT) and/or magnetic resonance imaging (MRI) who had at least 1 year of follow-up were evaluated retrospectively. Results: A total of 78 patients underwent follow-up for an average of 55.7 months. Most patients were asymptomatic, and 35 (37.2\%) showed complete resolution. The initial mean PCL size was $1.6 \pm 0.9 \mathrm{~cm}$ (range, 0.5 to $5.6 \mathrm{~cm}$ ). The average time to initial decrease in size and complete resolution of PCLs were 32.1 and 41.5 months, respectively. Compared with PCLs that completely resolved, presence of underlying malignancy was associated with partial resolution of PCLs in multivariable analysis (hazard ratio, 0.51; 95\% confidence interval, 0.32 to $0.81 ; p=0.005$ ). Endoscopic ultrasound (EUS) identified detailed findings, especially the presence of septum $(p<0.001)$, calcification $(p=0.015)$ and lobulation $(p=0.001)$ that were not found on CT/MRI. Conclusions: Asymptomatic small PCLs without high-risk stigmata can naturally decrease in size at approximately 3 years, and complete resolution can be expected in the absence of underlying malignancy. Regular follow-up of approximately 3 years with EUS may be a reasonable and safe alternative when planning the initial treatment of small PCLs without high-risk stigmata. (Gut Liver 2020;14:248-256)

Key Words: Pancreas; Cysts; Neoplasms; Time

\section{INTRODUCTION}

As a result of improved imaging technology, pancreatic cystic lesions (PCLs) are being detected with an increasing frequency. Incidental PCLs are found in 2.6\% of patients undergoing abdominal imaging performed for unrelated reasons, and the frequency increases with age, reaching $10 \%$ in those older than 70 years. ${ }^{1,2}$ Recent radiological studies have suggested that PCLs are identified in up to $15 \%$ of patients who underwent magnetic resonance imaging (MRI)., Because PCLs have a broad differential diagnosis, they often present a diagnostic and therapeutic challenge. The correct characterization of any PCL is a key point in determining appropriate management.

Surgical resection is generally recommended for symptomatic or premalignant PCLs or those that demonstrate possible malignancy based on imaging features and/or biopsy results. However, uncertainty remains about the effective long-term management for PCLs, which often do not have a definite diagnosis and have inadequate natural history data. Therefore, current consensus guidelines divide imaging characteristics into either "high-risk stigmata," which are absolute indications for operation, or "worrisome features," for which further evaluation such as cyst-fluid analysis and close follow-up is suggested., Finally, a decision analysis of PCLs without high-risk stigmata should be factored in the patient's age, cyst location, cyst size, and underlying disease to assess the most cost-effective approach for patients with these lesions.

To date, although a few studies have shown that some PCLs without high-risk stigmata may naturally decrease in size, the clinical features and natural course of shrinkage of PCLs are still under investigation. The primary objective of this study was to evaluate the timing of the initial decrease in size of PCLs. The

\footnotetext{
Correspondence to: Sung Koo Lee

Division of Gastroenterology, Department of Internal Medicine, Asan Medical Center, University of Ulsan College of Medicine, 88 Olympic-ro 43gil, Songpa-gu, Seoul 05505, Korea

Tel: +82-2-3010-3186, Fax: +82-2-476-0824, E-mail: sklee@amc.seoul.kr

Received on November 2, 2018. Revised on January 27, 2019. Accepted on March 4, 2019. Published online January 7, 2020. pISSN 1976-2283 eISSN 2005-1212 https://doi.org/10.5009/gnl18488

(a) This is an Open Access article distributed under the terms of the Creative Commons Attribution Non-Commercial License (http://creativecommons.org/licenses/by-nc/4.0) which permits unrestricted non-commercial use, distribution, and reproduction in any medium, provided the original work is properly cited.
} 
secondary objective was to evaluate the clinical features and determine the factors predicting complete resolution in patients with spontaneous decrease in size of PCLs without high-risk stigmata. In addition, we also identified endoscopic ultrasound (EUS) finding and evaluated the usefulness of EUS to obtain detailed information of small PCLs.

\section{MATERIALS AND METHODS}

\section{Patients and data collection}

Patients who were suspected of having PCLs that decreased in size between two examinations at the Asan Medical Center between January 2000 and December 2016 were identified using the institutional radiology coding system of the Picture and Archiving Computer System. The inclusion criteria were patient age $>20$ years and a diagnosis of decreased PCL size based on computed tomography (CT) and MRI/MR cholangiopancreatography (MRCP) results. Patients were excluded if there was (1) a follow-up duration of <1 year; (2) an insignificant decrease $(<30 \%)$ in PCL size; (3) history or current biochemical and/or clinical evidence of acute pancreatitis; (4) established chronic pancreatitis based on previous imaging evaluation; (5) a history of Von Hippel-Lindau disease or cystic fibrosis; (6) a history of polycystic kidney disease or polycystic liver disease; and (7) a history of abdominal trauma and pancreatic cancer.

In the enrolled patients, demographic and clinical data were analyzed retrospectively with a special focus on associated underlying malignancy. We also evaluated PCLs with respect to location in the pancreas, cyst size, cyst number, cyst morphology, and remarkable radiological features including the presence of a septum, calcification, cyst wall thickening, and solid component, as well as with respect to biochemical chemistry. In addition, the detailed imaging presented by EUS that were not seen on CT or MRI/MRCP were further evaluated. The study protocol was reviewed and approved by the Institutional Review Board of Asan Medical Center (IRB number: 2016-0864). Written informed consents were obtained.

\section{Definitions}

The size of the PCLs was defined as the largest diameter measured using CT and/or MRI/MRCP. If multiple PCLs were present, the largest diameter was used. PCLs located in the head, neck, and uncinate process of the pancreas were included into one category as head lesions. The shrinkage of the PCLs was considered significant if the decrease was $\geq 30 \%$ relative to the initial size. Resolution of PCLs was defined as the absence of imaging evidence of a remnant cyst. Worrisome features of PCLs were defined according to the Fukuoka criteria as follows: cyst size $\geq 3 \mathrm{~cm}$, thickened or enhancing cyst wall, pancreatic duct dilatation from 5 to $9 \mathrm{~mm}$, non-enhancing solid component, and change in pancreatic duct diameter with distal parenchyma atrophy. ${ }^{5}$ Lesions were defined as asymptomatic if they were identified during the evaluation for an alternate presenting symptom or in the absence of abdominal pain. The follow-up duration was recorded as the time in months from the initial detection of PCLs to the last available imaging data.

\section{Follow-up}

All enrolled patients received the first follow-up imaging study (CT or MRI/MRCP) from 3 to 6 months after the initial diagnosis of PCLs in order to obtain a perspective on the cyst growth rates. In case of a lack of significant changes in cyst size and features, the follow-up interval was extended to 6 months to 1 year according to the individual clinical situation. The choice of follow-up imaging modality (CT or MRI/MRCP) depended on baseline imaging modality. EUS was also performed in patients suspected of having worrisome features or with a change of clinical features.

\section{Statistical analyses}

Continuous variables are presented as mean \pm standard deviation and median (interquartile range), and compared using the Student t-test or Mann-Whitney U-test, depending on the data distribution. Categorical variables are reported as frequency or percentage and compared using the chi-square or Fisher exact test, as appropriate. The log-rank test was used to compare the curves of the decrease in size and complete resolution of PCLs obtained using the Kaplan-Meier survival analysis. Univariable and multivariable analyses were performed to evaluate the predictors of complete resolution of PCLs based on a Cox proportional hazards regression model, and $\mathrm{p}<0.05$ was considered to indicate a statistically significant difference. All statistical analyses were performed using PASW statistics version 18.0 (SPSS Inc., Chicago, IL, USA).

\section{RESULTS}

\section{Patient characteristics}

During the study period, 260 patients with spontaneous decrease PCLs size were identified, of whom 78 patients were enrolled. A total of 182 patients were excluded for the following reasons: pancreatitis $(\mathrm{n}=129)$, insignificant decrease in PCL size $(n=33),<1$ year of follow-up ( $n=6)$, Von Hippel-Lindau disease $(\mathrm{n}=3)$, pancreas divisum $(\mathrm{n}=3)$, concurrent pancreatic cancer $(\mathrm{n}=3)$, autoimmune pancreatitis $(\mathrm{n}=2)$, polycystic kidney disease/ polycystic liver disease $(n=2)$, and trauma $(n=1)$ (Fig. 1). The mean age of the patients in our study was $54.4 \pm 11.9$ years and 49 patients (62.8\%) were women. Overall, 70 patients (89.7\%) had asymptomatic lesions and six (7.7\%) had family history of pancreatic cancer. Among them, 17 (21.8\%) had a history of a previous malignancy or active disease, except for pancreatic cancer. A total of 27 patients (34.6\%) consumed alcohol on a regular basis and 13 (16.7\%) were smokers. The mean follow-up period was $55.7 \pm 37.1$ months. 


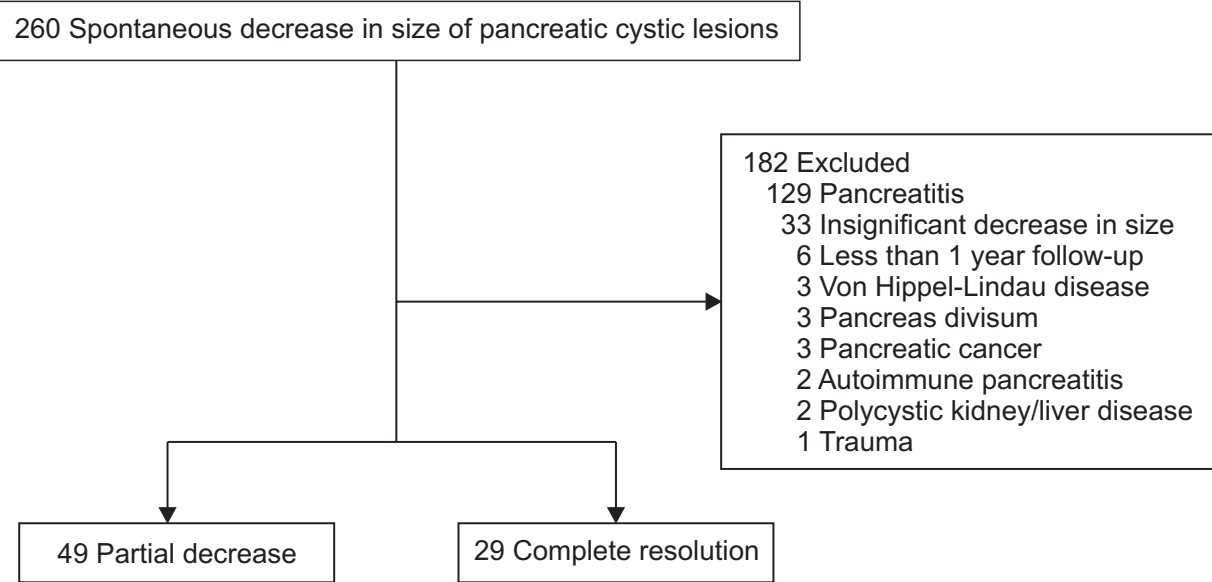

Fig. 1. Flow diagram of the study design.

Table 1. Baseline Characteristics of Patients with a Decrease in Size of Pancreatic Cystic Lesions

\begin{tabular}{lcccc}
\hline \multicolumn{1}{c}{ Variable } & Total $(\mathrm{n}=78)$ & Partial decrease $(\mathrm{n}=49)$ & Complete resolution $(\mathrm{n}=29)$ & $\mathrm{p}$-value \\
\hline Age, $\mathrm{yr}$ & $54.4 \pm 11.9$ & $57.5 \pm 12.1$ & $49.2 \pm 9.8$ & 0.003 \\
Female sex & $49(62.8)$ & $31(63.3)$ & $18(62.1)$ & 0.916 \\
BMI, $\mathrm{kg} / \mathrm{m}^{2}$ & $23.4 \pm 3.0$ & $23.5 \pm 2.4$ & $23.4 \pm 3.8$ & 0.896 \\
Malignancy & $17(21.8)$ & $15(30.6)$ & $2(6.9)$ & 0.014 \\
Family history of pancreatic cancer & $6(7.7)$ & $5(10.2)$ & $1(3.4)$ & 0.403 \\
Alcohol & $27(34.6)$ & $17(34.7)$ & $4(13.8)$ & 0.985 \\
Smoking & $13(16.7)$ & $9(18.4)$ & $28(96.6)$ & 0.757 \\
Asymptomatic & $70(89.7)$ & $42(85.7)$ & & 0.246 \\
Laboratory finding & & & $9.3 \pm 8.1$ & $63.9 \pm 25.3$ \\
$\quad$ CA19-9, U/mL & $11.8 \pm 17.5$ & $13.3 \pm 21.2$ & $32.5 \pm 9.0$ & 0.338 \\
Amylase, U/L & $66.3 \pm 24.8$ & $67.7 \pm 24.6$ & $6.2 \pm 2.3$ & 0.522 \\
Lipase, IU/L & $37.0 \pm 17.2$ & $39.5 \pm 19.9$ & $58.1 \pm 33.3$ & 0.061 \\
$\quad$ WBC, $10^{3} / \mu \mathrm{L}$ & $6.1 \pm 2.1$ & $6.1 \pm 1.9$ & & 0.757 \\
Mean follow-up, mo & $55.7 \pm 37.1$ & $54.2 \pm 39.4$ & & 0.655
\end{tabular}

Data are presented as mean \pm SD or number $(\%)$.

BMI, body mass index; CA19-9, carbohydrate antigen 19-9; WBC, white blood cell.

The detailed baseline characteristics of patients with decreased PCL size (partial decrease or complete resolution) are shown in Table 1 . The mean age and the presence of malignancy at the time of presentation were significantly higher in the partial decrease in size group than in the complete resolution group $(p=0.003$ and $p=0.014$, respectively). The other baseline characteristics of the patients did not differ between the two groups. The patient distribution and median size according to age of decreased PCLs is shown in Fig. 2.

\section{Total radiological characteristics of PCLs}

The radiological imaging modality most commonly used at initial assessment was abdominal CT (70.1\%) followed by MRI/ MRCP (32.9\%). Among the patients, 38 (48.7\%) underwent EUS at least once. The mean size of cysts on presentation was $1.6 \pm 0.9$ $\mathrm{cm}$ and the median size increased with age (Fig. 2). The initial cyst size was $<1 \mathrm{~cm}$ in 15 patients (19.2\%), $\geq 1$ and $<2 \mathrm{~cm}$ in 43 patients (55.1\%), and $\geq 2 \mathrm{~cm}$ in 20 patients (25.6\%). Most patients had a solitary (84.6\%) and round (71.8\%) cyst. Approximately half of the cysts were seen in the head (43.6\%), whereas others were found in the body (25.6\%), tail (19.2\%), and multifocal sites (11.5\%). At the initial diagnosis, there were 19 (24.4\%) patients with at least one worrisome features, including cyst size $\geq 3 \mathrm{~cm}(\mathrm{n}=8)$, non-enhancing solid component $(\mathrm{n}=5)$, thickened cyst wall $(n=1)$, change in pancreatic duct diameter with distal parenchyma atrophy $(n=1)$, and pancreatic duct dilatation $(n=5)$. Of the 78 patients, two (2.6\%) developed a new cyst during the follow-up period. The radiological characteristics of the patients according to the type of decrease in size are summarized in Table 2. The radiological characteristics except initial PCL size of the patients did not differ between the two groups. 


\section{Diagnostic value of EUS over CT/MRI in small PCLs}

EUS was performed in 38 patients (48.7\%) and identified 39 detailed findings of internal structures and morphology of small PCLs that were not found on CT/MRI. Septum was detected on

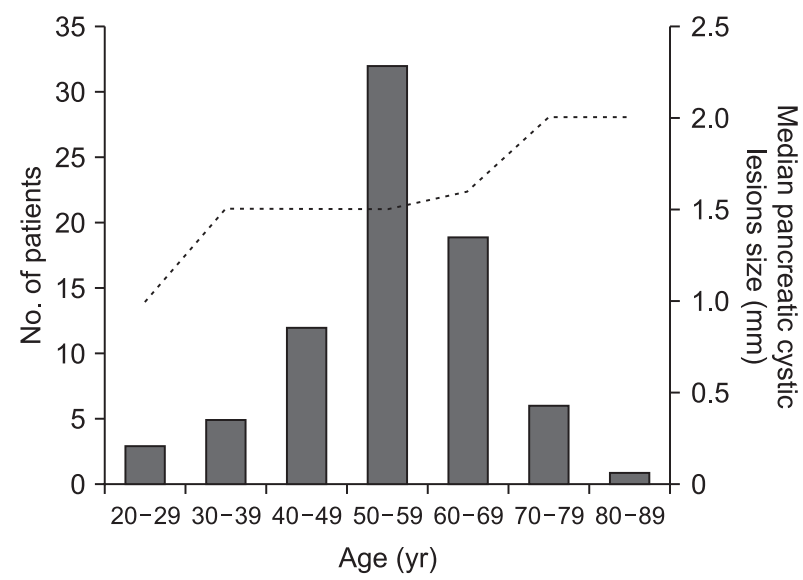

Fig. 2. Histogram showing the age distribution at presentation among the study subjects with a decrease in size of pancreatic cystic lesions. seven lesions by CT/MRI and 16 lesions by EUS (9.0\% vs $42.1 \%$ $\mathrm{p}<0.001)$. Calcification was detected on two lesions by CT/MRI and six lesions by EUS (2.6\% vs 15.8\%, p=0.015). Likewise, cystic lobulation was detected on 4 lesions by CT/MRI and 11 lesions by EUS (5.1\% vs 28.9\%, p=0.001). In addition, we have found additional findings of non-enhancing solid portion $(n=3)$, pancreatic duct communication $(\mathrm{n}=2)$, and thickened wall $(\mathrm{n}=1)$ by EUS (Table 3). However, these additional EUS findings did not show significant differences between partial decrease and complete resolution group.

\section{Timing of initial decrease in size and complete resolution of PCLs}

The mean interval between the first diagnosis and initial decrease in size was $32.1 \pm 34.7$ months, which was shorter in the complete resolution group than in the partial shrinkage group (39.7 \pm 5.6 months vs $20.9 \pm 3.9$ months, $\mathrm{p}=0.043$ ). In the complete resolution group, the mean interval between the first diagnosis and the complete resolution was $41.5 \pm 34.5$ months.

Table 2. Radiological Characteristics of Patients with a Decrease in the Size of PCLs

\begin{tabular}{|c|c|c|c|c|}
\hline Variable & Total $(n=78)$ & Partial decrease $(n=49)$ & Complete resolution $(n=29)$ & p-value \\
\hline Initial PCLs size, $\mathrm{cm}$ & $1.6 \pm 0.9(0.5-5.6)$ & $1.8 \pm 1.0(0.6-5.6)$ & $1.3 \pm 0.7(0.5-3.4)$ & 0.009 \\
\hline Location of PCLs & & & & 0.268 \\
\hline Head & $34(43.6)$ & $24(49.0)$ & $10(34.5)$ & \\
\hline Body & $20(25.6)$ & $11(22.4)$ & $9(31.0)$ & \\
\hline Tail & $15(19.2)$ & $7(14.3)$ & $8(27.6)$ & \\
\hline Multifocal & $9(11.5)$ & $7(14.3)$ & $2(6.9)$ & \\
\hline No. of PCLs & & & & 0.193 \\
\hline Single & $66(84.6)$ & 39 (79.6) & $27(93.1)$ & \\
\hline Multiple & $12(15.4)$ & $10(20.4)$ & $2(6.9)$ & \\
\hline Appearance & & & & 0.075 \\
\hline Round & $56(71.8)$ & $37(75.5)$ & $19(65.5)$ & \\
\hline Tubular & $7(9.0)$ & $6(12.2)$ & $1(3.4)$ & \\
\hline Lobular & 15 (19.2) & $6(12.2)$ & $9(31.0)$ & \\
\hline Worrisome features & $19(24.4)$ & 15 (30.6) & $4(13.8)$ & 0.094 \\
\hline$\geq 3 \mathrm{~cm}$ & $8(10.3)$ & $7(14.3)$ & $1(3.4)$ & 0.246 \\
\hline Non-enhancing solid portion & $5(6.4)$ & $4(8.2)$ & $1(3.4)$ & 0.646 \\
\hline Thickened wall & $1(1.3)$ & 0 & $1(3.4)$ & 0.372 \\
\hline P-duct change with distal parenchyma atrophy & $1(1.3)$ & $1(2.0)$ & 0 & 1.000 \\
\hline P-duct dilatation (5-9 mm) & $5(6.4)$ & $4(8.2)$ & $1(3.4)$ & 0.646 \\
\hline \multicolumn{5}{|l|}{ Other findings } \\
\hline P-duct communication & $10(12.8)$ & $8(16.3)$ & $1(3.4)$ & 0.142 \\
\hline Calcification & $8(10.3)$ & $3(6.1)$ & $5(17.2)$ & 0.140 \\
\hline Septum & $23(29.5)$ & $16(32.7)$ & $7(24.1)$ & 0.425 \\
\hline Newly developed cyst & $2(2.6)$ & $1(2.0)$ & $1(3.4)$ & 1.000 \\
\hline
\end{tabular}

Data are presented as mean \pm SD (range) or number (\%).

PCLs, pancreatic cystic lesions; P-duct, pancreatic duct. 
Table 3. Results of New Additional EUS Findings Compared with the CT/MRI Findings Regarding Small Pancreatic Cystic Lesions

\begin{tabular}{llrr}
\hline \multicolumn{1}{c}{ Variable } & $\begin{array}{c}\text { CT/MRI } \\
(\mathrm{n}=78)\end{array}$ & $\begin{array}{c}\text { EUS } \\
(\mathrm{n}=38)\end{array}$ & p-value \\
\hline Non-enhancing solid portion & $2(2.6)$ & $3(7.9)$ & 0.329 \\
Thickened wall & 0 & $1(2.6)$ & 0.328 \\
P-duct communication & $8(10.3)$ & $2(5.3)$ & 0.494 \\
Septum & $7(9.0)$ & $16(42.1)$ & $<0.001$ \\
Calcification & $2(2.6)$ & $6(15.8)$ & 0.015 \\
Lobulation & $4(5.1)$ & $11(28.9)$ & 0.001 \\
\hline
\end{tabular}

Data are presented as number (\%).

EUS, endoscopic ultrasound; CT, computed tomography; MRI, magnetic resonance imaging; P-duct, pancreatic duct.

\section{Predictive factors for complete resolution of PCLs}

Compared with patients with partial decrease in the size of PCLs, those with complete resolution of PCLs were younger and had significantly lower presence of malignancy. Univariable analysis revealed an association between complete resolution and age (hazard ratio [HR], 0.97; 95\% confidence interval [CI], 0.94 to $0.99 ; p=0.028$ ). Likewise, the presence of malignancy was significantly associated with complete resolution (HR, 4.66; 95\% CI, 1.11 to $19.65 ; \mathrm{p}=0.036$ ). In addition, initial PCL size was significantly associated with complete resolution (HR, 0.54; 95\% CI, 0.29 to $0.99 ; p=0.049$ ). The results of the multivariable analysis revealed that presence of malignancy was the only significant independent factor predictive of complete resolution in these patients (HR, 0.15; 95\% CI, 0.32 to 0.73 ; $p=0.018$ ) (Table 4).

Table 4. Results of Univariable and Multivariable Analyses for the Complete Resolution of PCLs Based on Cox Proportional Hazards Regression Analysis

\begin{tabular}{|c|c|c|c|c|}
\hline \multirow{2}{*}{ Variable } & \multicolumn{2}{|c|}{ Univariable } & \multicolumn{2}{|c|}{ Multivariable } \\
\hline & HR $(95 \%$ CI) & $\mathrm{p}$-value & HR $(95 \%$ CI) & p-value \\
\hline Age & $0.97(0.94-0.99)$ & 0.028 & & \\
\hline Male sex & $1.13(0.53-2.43)$ & 0.757 & & \\
\hline Family history of pancreatic cancer & $1.98(0.267-14.69)$ & 0.505 & & \\
\hline Symptoms & $3.06(0.48-26.81)$ & 0.211 & & \\
\hline Malignancy & $4.66(1.11-19.65)$ & 0.036 & $0.15(0.32-0.73)$ & 0.018 \\
\hline Alcohol & $1.22(0.55-2.67)$ & 0.625 & & \\
\hline Smoking & $0.89(0.31-2.59)$ & 0.830 & & \\
\hline Lipase & $0.97(0.94-1.01)$ & 0.112 & & \\
\hline Initial PCLs size & $0.54(0.29-0.99)$ & 0.049 & & \\
\hline \multicolumn{5}{|l|}{ Cyst location } \\
\hline Head & 1 (Reference) & & & \\
\hline Body & $1.38(0.29-6.42)$ & 0.677 & & \\
\hline Tail & $2.85(0.61-13.49)$ & 0.185 & & \\
\hline Multifocal & $3.40(0.71-16.35)$ & 0.127 & & \\
\hline Multiple cyst & $3.73(0.87-15.57)$ & 0.075 & & \\
\hline \multicolumn{5}{|l|}{ Appearance } \\
\hline Round & 1 (Reference) & & & \\
\hline Tubular & $0.68(0.30-1.52)$ & 0.349 & & \\
\hline Lobular & $0.19(0.02-1.50)$ & 0.115 & & \\
\hline \multicolumn{5}{|l|}{ Worrisome features } \\
\hline$\geq 3 \mathrm{~cm}$ & $2.01(0.27-15.02)$ & 0.496 & & \\
\hline Non-enhancing solid portion & $4.98(0.61-40.37)$ & 0.133 & & \\
\hline Thickened wall & $0.64(0.09-4.86)$ & 0.673 & & \\
\hline P-duct change with distal parenchyma atrophy & $20.81(0.00-9,022.80)$ & 0.647 & & \\
\hline P-duct dilatation (5-9 mm) & $2.83(0.38-21.28)$ & 0.312 & & \\
\hline P-duct communication & $6.59(0.84-51.25)$ & 0.072 & & \\
\hline Calcification & $0.64(0.24-1.68)$ & 0.362 & & \\
\hline Septum & $1.55(0.66-3.63)$ & 0.316 & & \\
\hline Newly developed cyst & $1.42(0.19-10.62)$ & 0.732 & & \\
\hline
\end{tabular}

HR, hazard ratio; CI, confidence interval; PCLs, pancreatic cystic lesions; P-duct, pancreatic duct. 


\section{Patterns of initial decrease in size and complete resolu- tion of PCLs according to presence of malignancy}

The interval between the first diagnosis and initial decrease in PCL size tended to increase with the presence of malignancy in the Kaplan-Meier curve. When there was no malignancy, the mean initial decrease in the size interval was $31.5 \pm 4.8$ months (95\% CI, 22.1 to 40.8). When malignancy was present the interval increased to $34.0 \pm 5.9$ months (95\% CI, 22.3 to 45.7 ); however, there was no statistical difference in the log-rank test ( $p=0.654$ ). In contrast, the maintenance period of remnant PCLs showed an increasing pattern as the presence of the malignancy. When there was no malignancy, the mean maintenance period of remnant PCLs was 92.7 \pm 14.3 months (95\% CI, 64.7 to 120.8), which was significantly shorter than that when malignancy was presented (133.1 \pm 10.9 ; 95\% CI, 111.8 to $154.4 ; \mathrm{p}=0.020)$ (Fig. 3).

\section{DISCUSSION}

There is scarce information about the natural history of the spontaneous decrease in the size of PCLs without high-risk stigmata in patients undergoing surveillance. Although a few studies have shown the frequency of spontaneous decrease in PCL size, the timing of the decrease in size and the clinical features of this shrinkage are largely unknown. To our knowledge, this is the first study to present the timing of the initial decrease in size, as well as the significance of the underlying malignancy, contributing to the prognosis of PCLs.

Some PCLs show spontaneous resolution (partial or complete), with a frequency of $7 \%$ to $36 \% .^{7-9}$ Although most of these lesions are asymptomatic small cysts and the definition of decrease in PCL size differs, PCLs could decrease in size or even show complete resolution without any intervention in some cases. In this study, 78 patients showed a decrease in the size of PCLs, which naturally began to decrease in size at approximate- ly 3 years after the diagnosis, and 37.2\% of these patients (29/78) showed complete resolution, even with a maximum diameter of $3.4 \mathrm{~cm}$. These findings suggest that not only small PCLs but also some large PCLs can decrease in size, and once they begin to decrease, more than one-third of these lesions may completely resolve.

The safety of a follow-up approach to indeterminate PCLs without high-risk stigmata remains controversial, as the natural history of PCLs is largely unknown. The 2012 Fukuoka consensus guidelines recommend operation in patients with symptoms and high-risk stigmata owing to their higher risk of malignancy. ${ }^{5}$ In patients with worrisome features, further evaluation with EUS is recommended to investigate for the definite presence of a mural nodule, main duct involvement, or cytology that suggests malignancy. ${ }^{3,5}$ In the evaluation of the impact of worrisome features on complete resolution, although the overall or individual prevalence of worrisome features was higher in the partial shrinkage group than in the complete resolution group, there was no significant difference between the two groups. However, our study suggests that even in patients with worrisome features, the size of the PCLs could decrease or the lesions could completely disappear. Small PCLs with a tubular shape, a septum, and a prominent pancreatic duct are more likely to progress. ${ }^{10}$ Therefore, they were not related to the complete resolution of PCLs in our study. Similarly, other imaging features, such as the size, location, and number and morphology of PCLs were also not related to complete resolution.

According to recent studies, EUS showed superiority in the diagnosis of small PCLs, for its high spatial resolution which can identified the internal structures such as septum and mural nodules. ${ }^{11,12}$ EUS was superior to CT/MRI in the characterization of small PCLs, precisely characterizing internal structure; septum $(\mathrm{p}=0.004)$ and mural nodule $(\mathrm{p}=0.028){ }^{12}$ Likewise, we also described the value of EUS in the characterization of small PCLs over CT/MRI. We performed EUS in only about half of the pa-
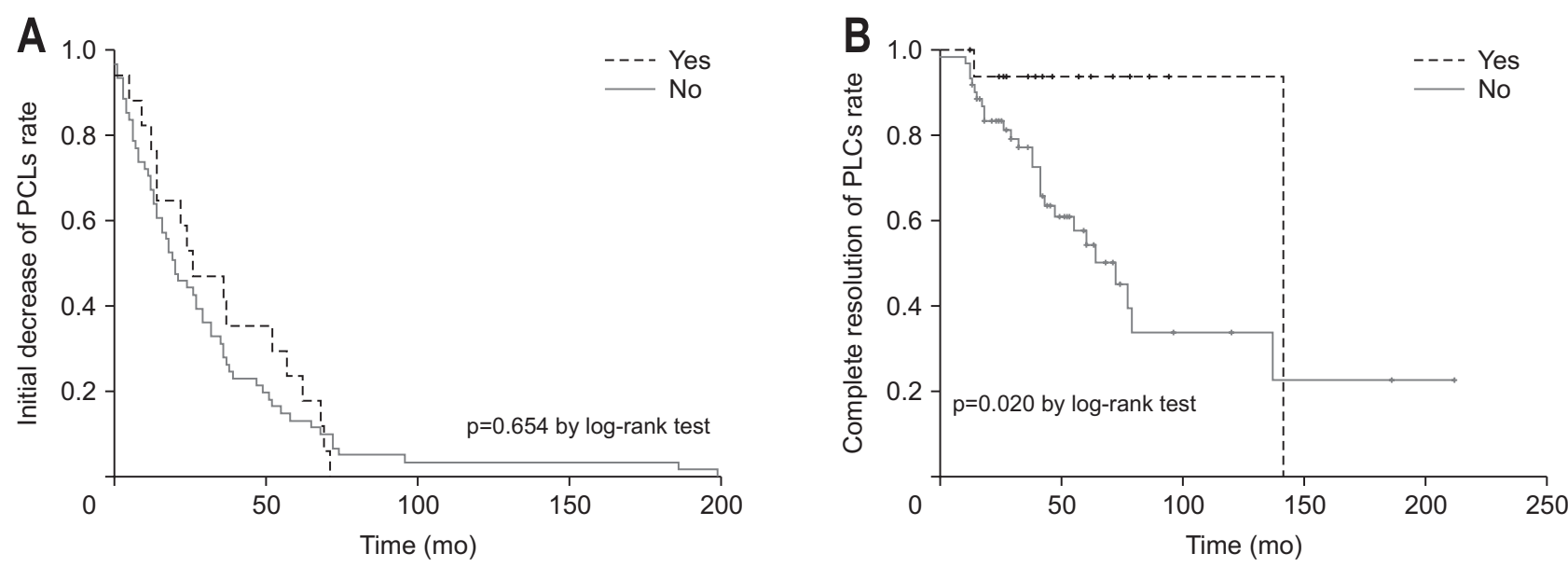

Fig. 3. Kaplan-Meier curve of the (A) initial decrease and (B) complete resolution rate of pancreatic cystic lesions (PCLs) according to the presence of an underlying malignancy. 
tients, however, we found that it is more sensitive to identifying internal structures; septum $(\mathrm{p}<0.001)$ and calcification $(\mathrm{p}=0.015)$. In addition, EUS provides more detailed morphological feature, even small cystic lobulation. Based on these EUS findings, it is possible that the majority of these PCLs are serous cystadenoma (Fig. 4). However, even in small asymptomatic lesions, EUS can help to characterize small PCLs and provide detailed information to understand the natural history of spontaneous decreased in size of PCLs.

Recent studies have questioned whether PCLs are associated with underlying systemic disorders. ${ }^{13,14}$ PCL is a disease of the elderly; it occurs in older patients, and advanced age proved to be a significant factor correlated to PCLs. ${ }^{15}$ Its prevalence increases with patient age, and the cumulative prevalence is as high as $40 \%$ in patients older than 70 years. Moreover, increased patient age correlate most strongly with decreased overall survival. ${ }^{16}$ In addition, PCLs can occur as an extrarenal complication in patients with cystic lesions of the liver and kidney. ${ }^{17}$ Another analysis revealed that the prevalence of PCLs was significantly higher in patients undergoing hemodialysis (odds ratio, 6.38; 95\% CI, 4.82 to 8.48) than in controls (9.3\% vs $1.3 \%, \mathrm{p}<0.001){ }^{18}$ Moreover, patients with intraductal papillary mucinous neoplasm (IPMN) had a higher prevalence of extrapancreatic malignancies and benign neoplasms, including colon polyps. ${ }^{19}$ In previous reports, patients with IPMN had a high observed/expected ratio for colorectal carcinoma (2.26; 95\% CI, 1.17 to 3.96$)$ and the most frequent benign neoplasm was colonic polyp (114/471). ${ }^{20,21}$ Therefore, the authors suggested that it is necessary to screen patients with IPMN for extrapancreatic malignancy. In our study, we also studied the clinical factors predictive of complete resolution in patients with a spontaneous decrease in PCL size and revealed that the underlying malignancy seems to predict a high frequency of resolution of PCLs. A total of 17 patients (21.8\%) had extrapancreatic malignancy, most commonly in the stomach $(n=7)$, followed by the liver $(n=4)$ and the thyroid $(n=2)$. The number of patients with a history of or a current malignancy was significantly lower in the complete resolution group ( 15 vs $2, \mathrm{p}=0.014$ ). On the basis of our data, the underlying malignancy state may affect not only the development but also the resolution of PCLs.

Our study has several limitations. The principal limitation of our study is the lack of a pathological diagnosis of PCLs. We excluded patients with pseudocyst; however, we could not completely exclude those with benign cysts such as lymphangioma, lymphoepithelial, and retention cysts. The key factor in determining the treatment and prognosis of PCLs is the tissue result, however, establishing a definitive diagnosis of PCLs was usually impossible because of refusal to invasive procedure in patients with spontaneous decreased in size of PCLs and the observational nature in our study. Another limitation is the older imaging technology, with the CT, MRI/MRCP, and EUS equipment from a maximum of 16 years ago being inferior to the presently available imaging devices. With the development of modern imaging modalities, more and smaller lesions were likely to have been detected in the more recent study period. In particular, the imaging modality associated with detecting worrisome features may have a different sensitivity between the previous and the current testing. In addition, because MRI/ MRCP and EUS were not performed in all patients, it is possible that worrisome features were underdetected. Finally, our study only included patients with spontaneous decrease in size of PCLs, therefore, did not provide the natural course and clinical
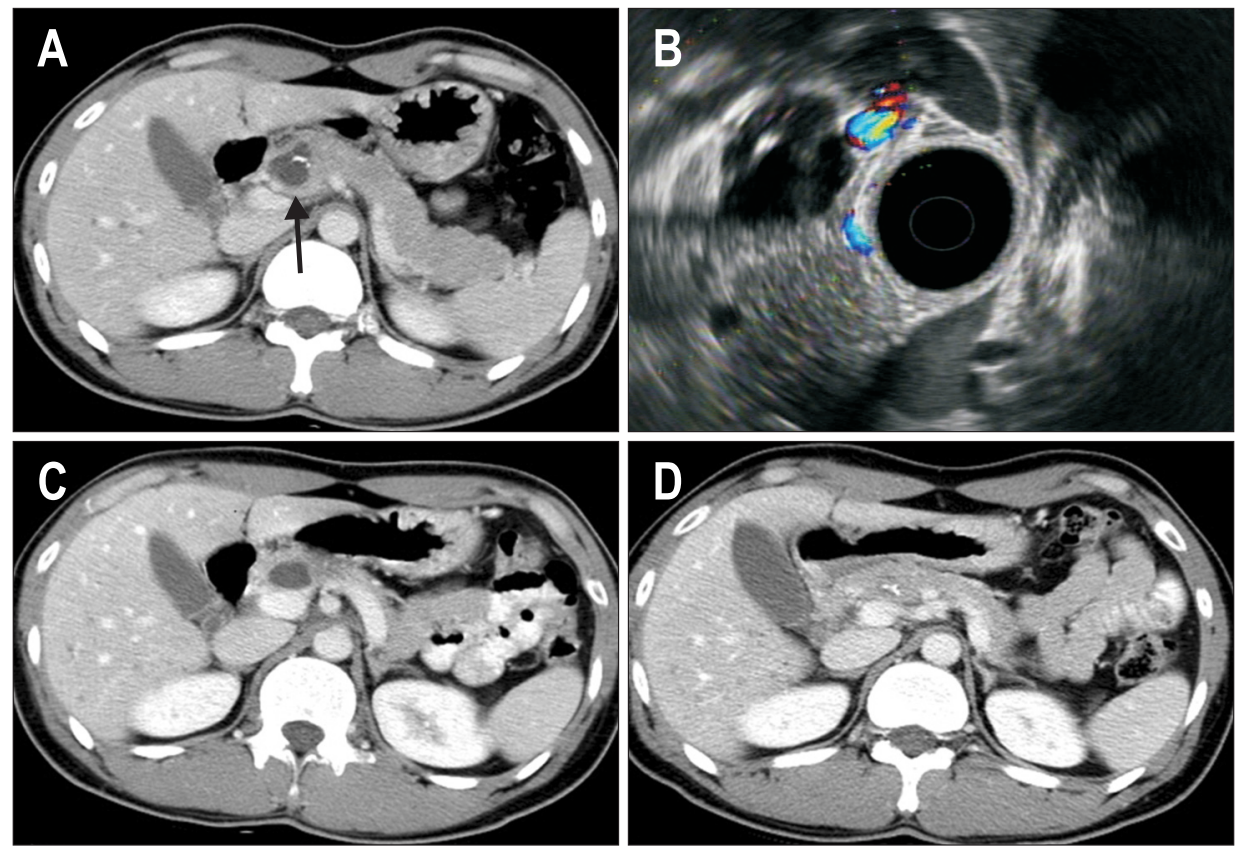

Fig. 4. A 39-year-old man with an incidentally identification of a 2-cm pancreatic cystic lesion (PCL) in the pancreatic head. (A) Initial computed tomography (CT) showed a mildly lobulated contour and internal calcification in the PCL (arrow). (B) Endoscopic ultrasound also showed a lobulated contour and septa and calcification in the PCL. (C) After 2 years, the size of PCL tended to be slightly smaller on CT. (D) After 4 years, the PCL seen on previous CT is not visible, and slight calcification remains at the site of the previous PCL. 
features of patients with PCLs that have increased or not change in size. Further comparative studies between spontaneous decreased, unchanged, and increased in size of PCLs are necessary to standardize these results.

In conclusion, our study showed that asymptomatic small PCLs spontaneously decreased in size at approximately 3 years after the diagnosis, and about one-third of patients with these lesions experienced complete resolution, according to the presence of malignancy. EUS helps characterize the features of small PCLs that were not found in CT/MRI and provides more detailed information, especially internal structures and small morphological features. Regular follow-up of about 3 years with EUS may be a reasonable and safe alternative when planning the initial treatment of small PCLs without high-risk stigmata and may help avoid unnecessary invasive diagnostic procedures or therapeutic intervention including surgery.

\section{CONFLICTS OF INTEREST}

No potential conflict of interest relevant to this article was reported.

\section{AUTHOR CONTRIBUTIONS}

Study concept and design, drafting of the manuscript: H.W.L., S.K.L. Acquisition of data and result interpretation and discussion: J.H.J., H.W.L. Acquisition of data and technical and material support: T.J.S., D.H.P., S.S.L. Critical revision of the manuscript for important intellectual content: D.W.S., M.H.K., S.K.L. All authors contributed to critical revision and approved of the final version.

\section{ORCID}

$\begin{array}{ll}\text { Hyun Woo Lee } & \text { https://orcid.org/0000-0001-9197-5783 } \\ \text { Sung Koo Lee } & \text { https://orcid.org/0000-0003-4155-4416 } \\ \text { Jae Hyuck Jun } & \text { https://orcid.org/0000-0002-8005-8215 } \\ \text { Tae Jun Song } & \text { https://orcid.org/0000-0002-6156-8746 } \\ \text { Do Hyun Park } & \text { https://orcid.org/0000-0002-0270-6969 } \\ \text { Sang Soo Lee } & \text { https://orcid.org/0000-0002-3785-2341 } \\ \text { Dong-Wan Seo } & \text { https://orcid.org/0000-0001-7525-8151 } \\ \text { Myung-Hwan Kim } & \text { https://orcid.org/0000-0003-0992-6734 }\end{array}$

\section{REFERENCES}

1. Laffan TA, Horton KM, Klein AP, et al. Prevalence of unsuspected pancreatic cysts on MDCT. AJR Am J Roentgenol 2008;191:802807.

2. de Jong K, Nio CY, Mearadji B, et al. Disappointing interobserver agreement among radiologists for a classifying diagnosis of pancreatic cysts using magnetic resonance imaging. Pancreas 2012;41:278-282.
3. Vege SS, Ziring B, Jain R, Moayyedi P; Clinical Guidelines Committee; American Gastroenterology Association. American gastroenterological association institute guideline on the diagnosis and management of asymptomatic neoplastic pancreatic cysts. Gastroenterology 2015;148:819-822.

4. Lee KS, Sekhar A, Rofsky NM, Pedrosa I. Prevalence of incidental pancreatic cysts in the adult population on MR imaging. Am J Gastroenterol 2010;105:2079-2084.

5. Tanaka M, Fernández-del Castillo C, Adsay V, et al. International consensus guidelines 2012 for the management of IPMN and MCN of the pancreas. Pancreatology 2012;12:183-197.

6. Tanaka M, Chari S, Adsay V, et al. International consensus guidelines for management of intraductal papillary mucinous neoplasms and mucinous cystic neoplasms of the pancreas. Pancreatology 2006;6:17-32.

7. Aranha GV, Prinz RA, Esguerra AC, Greenlee HB. The nature and course of cystic pancreatic lesions diagnosed by ultrasound. Arch Surg 1983;118:486-488.

8. Brook OR, Beddy P, Pahade J, et al. Delayed growth in incidental pancreatic cysts: are the current American college of radiology recommendations for follow-up appropriate? Radiology 2016;278:752-761.

9. Handrich SJ, Hough DM, Fletcher JG, Sarr MG. The natural history of the incidentally discovered small simple pancreatic cyst: longterm follow-up and clinical implications. AJR Am J Roentgenol 2005;184:20-23.

10. Tsai HM, Chuang CH, Shan YS, Liu YS, Chen CY. Features associated with progression of small pancreatic cystic lesions: a retrospective study. World J Gastroenterol 2015;21:13309-13315.

11. Sahani DV, Kadavigere R, Saokar A, Fernandez-del Castillo C, Brugge WR, Hahn PF. Cystic pancreatic lesions: a simple imagingbased classification system for guiding management. Radiographics 2005;25:1471-1484.

12. Lu X, Zhang S, Ma C, Peng C, Lv Y, Zou X. The diagnostic value of EUS in pancreatic cystic neoplasms compared with CT and MRI. Endosc Ultrasound 2015;4:324-329.

13. Wilcox CM. Editorial: management of the small asymptomatic pancreatic cyst: somehow along the way we forgot about the patient. Am J Gastroenterol 2017;112:1337-1339.

14. Patel NC, Palmer WC, Gill KR, et al. Association of intraductal papillary mucinous neoplasm (IPMN) with extra-pancreatic cystic lesions: is there a systemic cystic disorder? Am J Gastroenterol 2012;107:1265-1266

15. Sey MS, Teagarden S, Settles D, et al. Prospective cross-sectional study of the prevalence of incidental pancreatic cysts during routine outpatient endoscopic ultrasound. Pancreas 2015;44:11301133.

16. Cadili A, Bazarrelli A, Garg S, Bailey R. Survival in cystic neoplasms of the pancreas. Can J Gastroenterol 2009;23:537-542.

17. Sato Y, Mukai M, Sasaki M, et al. Intraductal papillary-mucinous neoplasm of the pancreas associated with polycystic liver and kidney disease. Pathol Int 2009;59:201-204. 
18. Ishikawa T, Takeda K, Itoh M, et al. Prevalence of pancreatic cystic lesions including intraductal papillary mucinous neoplasms in patients with end-stage renal disease on hemodialysis. Pancreas 2009;38:175-179.

19. Benarroch-Gampel J, Riall TS. Extrapancreatic malignancies and intraductal papillary mucinous neoplasms of the pancreas. World J Gastrointest Surg 2010;2:363-367.

20. Larghi A, Panic N, Capurso G, et al. Prevalence and risk factors of extrapancreatic malignancies in a large cohort of patients with intraductal papillary mucinous neoplasm (IPMN) of the pancreas. Ann Oncol 2013;24:1907-1911.

21. Reid-Lombardo KM, Mathis KL, Wood CM, Harmsen WS, Sarr MG. Frequency of extrapancreatic neoplasms in intraductal papillary mucinous neoplasm of the pancreas: implications for management. Ann Surg 2010;251:64-69. 